\title{
Flavitalea populi gen. nov., sp. nov., isolated from soil of a Euphrates poplar (Populus euphratica) forest
}

\author{
Correspondence \\ Chengxiang Fang \\ cxfang@whu.edu.cn \\ Fang Peng \\ fangpeng2@yahoo.com.cn
}

\author{
Yang Wang, ${ }^{1}$ Feng Cai, ${ }^{1}$ Yali Tang, ${ }^{2}$ Jun Dai, ${ }^{1}$ Huan Oi, ${ }^{1}$ Erkin Rahman, ${ }^{3}$ \\ Fang Peng ${ }^{1}$ and Chengxiang Fang ${ }^{1}$ \\ ${ }^{1}$ China Center for Type Culture Collection (CCTCC), College of Life Sciences, Wuhan University, \\ Wuhan 430072, PR China \\ ${ }^{2}$ Institute of Hydrobiology, Jinan University, Guangzhou 510632, PR China \\ ${ }^{3}$ College of Life Science and Technology, Xinjiang University, Urumchi 830046, PR China
}

\begin{abstract}
A novel strain, designated $\mathrm{HY}-50 \mathrm{R}^{\top}$, isolated from soil of a Euphrates poplar (Populus euphratica) forest in Xinjiang, China, was characterized using a polyphasic taxonomic approach. Cells of the isolate were Gram-reaction-negative, strictly aerobic, rod-shaped, non-motile, oxidase-negative and catalase-positive. Phylogenetic analysis based on 16S rRNA gene sequences showed that the isolate was a member of the phylum Bacteroidetes, its closest relatives being Niastella populi THYL- $44^{\top}$ (93.6\% similarity), Flavisolibacter ginsengisoli Gsoil $643^{\top}(93.5 \%)$, Terrimonas ferruginea IAM $15098^{\top}(93.3 \%)$ and Flavisolibacter ginsengiterrae Gsoil $492^{\top}(93.2 \%)$. The major fatty acids were iso- $C_{15: 1} G(11.7 \%)$, iso- $C_{15: 0}(19.6 \%)$ and iso- $\mathrm{C}_{17: 0} 3-\mathrm{OH}(19.3 \%)$. The predominant menaquinone of strain $\mathrm{HY}-50 \mathrm{R}^{\mathrm{T}}$ was MK-7 and the genomic DNA $\mathrm{G}+\mathrm{C}$ content was $46.8 \mathrm{~mol} \%$. Flexirubin-type pigments were not produced. Based on phylogenetic evidence and the results of phenotypic, genotypic and chemotaxonomic analysis, strain $\mathrm{HY}-50 \mathrm{R}^{\top}$ represents a novel species of a novel genus, for which the name Flavitalea populi gen. nov., sp. nov. is proposed. The type strain is $\mathrm{HY}^{-50 \mathrm{R}^{\top}}(=\mathrm{CCTCC} \mathrm{AB}$ $208255^{\top}=$ NRRL $B-59222^{\top}$ ).
\end{abstract}

The phylum Bacteroidetes (Garrity \& Holt, 2001) is phenotypically diverse and is still undergoing considerable taxonomic expansion and organization. Recently, the family Chitinophagaceae, belonging to the phylum Bacteroidetes, was established to incorporate several genera on the basis of $16 \mathrm{~S}$ rRNA gene sequence analyses and some phenotypic similarities (Kämpfer et al., 2011). The family Chitinophagaceae encompasses 15 genera, Balneola (Urios et al., 2006), Chitinophaga (Kämpfer et al., 2006), Ferruginibacter (Lim et al., 2009), Filimonas (Shiratori et al., 2009), Flavihumibacter (Zhang et al., 2010), Flavisolibacter (Yoon \& Im, 2007), Gracilimonas (Choi et al., 2009), Hydrotalea (Kämpfer et al., 2011), Lacibacter (Qu et al., 2009), Niabella (Kim et al., 2007), Niastella (Weon et al., 2006), Parasegetibacter (Zhang et al., 2009), Sediminibacterium (Qu \& Yuan, 2008), Segetibacter (An et al., 2007) and Terrimonas (Xie \& Yokota, 2006). During the course of

The GenBank/EMBL/DDBJ accession number for the 16S rRNA gene sequence of strain $\mathrm{HY}-50 \mathrm{R}^{\top}$ is $\mathrm{HM} 130561$.

One supplementary figure is available with the online version of this paper. a study on the diversity of bacterial communities associated with soil from a Euphrates poplar forest in Xinjiang, China, a bacterial strain, designated $\mathrm{HY}-50 \mathrm{R}^{\mathrm{T}}$, which formed yellow-pigmented colonies, was isolated. Comparative analysis of $16 \mathrm{~S}$ rRNA gene sequences suggested that strain $\mathrm{HY}-50 \mathrm{R}^{\mathrm{T}}$ was most closely related to members of the family Chitinophagaceae within the phylum Bacteroidetes. The strain was, therefore, subjected to further taxonomic investigation, the results of which suggest that strain HY$50 \mathrm{R}^{\mathrm{T}}$ represents a novel species of a novel genus in the family Chitinophagaceae.

To isolate the strain, a soil sample was serially diluted with sterilized water and the dilutions were plated onto R2A agar (Difco) and incubated at $30{ }^{\circ} \mathrm{C}$ for 4 days. Growth of strain $\mathrm{HY}-50 \mathrm{R}^{\mathrm{T}}$ was also evaluated on nutrient agar (NA, Difco), tryptic soy agar (TSA, Difco), $0.1 \times$ TSA and MacConkey agar (Difco) at $30^{\circ} \mathrm{C}$. As strain HY-50R ${ }^{\mathrm{T}}$ grew well on NA, R2A, TSA and $0.1 \times$ TSA, it was routinely cultured on $0.1 \times$ TSA at $30{ }^{\circ} \mathrm{C}$.

Colony morphology was observed visually and cell morphology and motility were observed using phase-contrast 
microscopy (Olympus BX51) using two-day-old cultures grown on $0.1 \times$ TSA at $30{ }^{\circ} \mathrm{C}$. Gram-staining was performed using the modified Hucker method (Gerhardt et al., 1994). Oxidase activity was evaluated via the oxidation of $1 \%$ $p$-aminodimethylaniline oxalate. Catalase activity was determined by measuring bubble production after the addition of $3 \%(\mathrm{v} / \mathrm{v})$ hydrogen peroxide solution. Tests for the hydrolysis of starch $(1 \%, \mathrm{w} / \mathrm{v})$, cellulose $(0.1 \%, \mathrm{w} / \mathrm{v})$, chitin from crab shells $(1 \%, \mathrm{w} / \mathrm{v})$ and casein $(5 \%, \mathrm{w} / \mathrm{v})$ were carried out as described by Smibert \& Krieg (1994). Growth under anaerobic conditions was determined by incubation at $30{ }^{\circ} \mathrm{C}$ on $0.1 \times$ TSA for 10 days in a GasPak (BBL) jar. Growth at $4,10,15,30,37$ and $42{ }^{\circ} \mathrm{C}$ was tested on $0.1 \times$ TSA. Growth at different $\mathrm{pH}$ levels was determined on $0.1 \times$ TSA adjusted to $\mathrm{pH} 4-10$ at intervals of $1 \mathrm{pH}$ unit before sterilization (Xu et al., 2005). Tolerance of $\mathrm{NaCl}$ was determined on $0.1 \times$ TSA supplemented with $1-5 \%(\mathrm{w} / \mathrm{v}) \mathrm{NaCl}$ at intervals of $1 \%$. The presence of flexirubin-type pigments was tested spectrophotometrically as described previously (Güde, 1980) using $20 \% \mathrm{KOH}$. Enzyme activities and biochemical characteristics were determined by using API 20 NE, API 20 E, API ZYM and API ID 32 GN test kits (bioMérieux) according to the manufacturer's instructions.

Menaquinones were extracted from lyophilized cells and identified using the method described by Xie \& Yokota (2003) using HPLC (UltiMate 3000, Dionex). Polyamines were analysed as described previously (Schenkel et al., 1995). The cellular fatty acids of strain $H Y-50 R^{T}$ and closely related type strains were saponified, methylated and extracted from cells grown on $\mathrm{R} 2 \mathrm{~A}$ at $30{ }^{\circ} \mathrm{C}$, according to the protocol of the Sherlock microbial identification system (MIDI, 1999), and analysed using GC (Agilent 6890).

Genomic DNA was prepared according to the method of Wilson (1987). The DNA G $+\mathrm{C}$ content was determined by HPLC (Mesbah et al., 1989). The 16S rRNA gene of strain HY-50 $\mathrm{R}^{\mathrm{T}}$ was amplified by PCR using an established method (Lane, 1991) and the product was sequenced (Invitrogen). 16S rRNA gene sequences of closely related species were retrieved from the EzTaxon database (Chun et al., 2007) and the sequences were aligned with the CLUSTAL_X program (Thompson et al., 1997). Phylogenetic analysis of $16 \mathrm{~S}$ rRNA gene sequences was performed using MEGA version 4.0 software (Tamura et al., 2007). Phylogenetic distances were calculated according to the neighbour-joining method (Saitou \& Nei, 1987), using the Kimura two-parameter model (Kimura, 1980), and the maximum-parsimony method (Fitch, 1971), using the close-neighbour-interchange algorithm (search level=2, random additions $=100$ ). Each resultant tree was evaluated by bootstrap analysis on the basis of 1000 replications (Felsenstein, 1985).

The almost-complete 16S rRNA gene sequence of strain $\mathrm{HY}-50 \mathrm{R}^{\mathrm{T}}$, comprising a continuous stretch of $1434 \mathrm{nt}$, was determined and compared with sequences of representatives of the phylum Bacteroidetes. Sequence similarity calculations performed after a neighbour-joining analysis

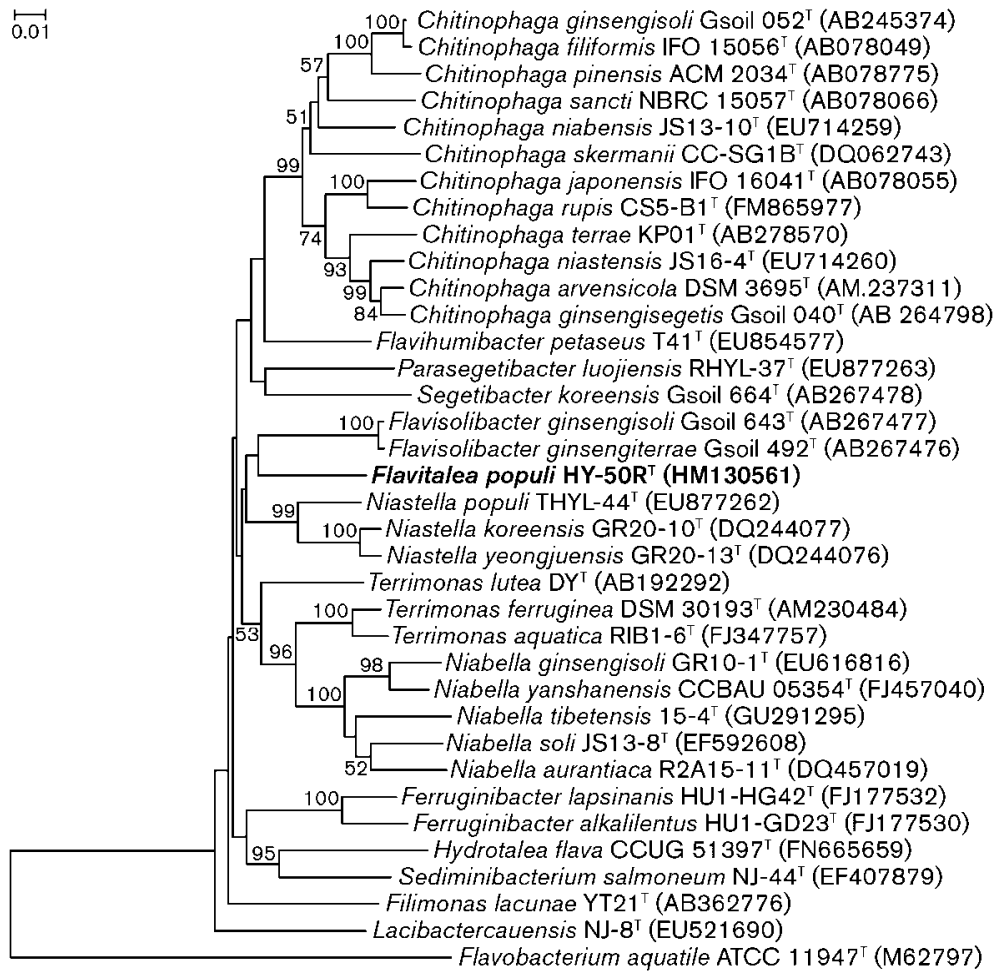

Fig. 1. Neighbour-joining phylogenetic tree based on 16S rRNA gene sequences of strain $\mathrm{HY}-50 \mathrm{R}^{\top}$ and representative members of the phylum Bacteroidetes. The tree was based on a comparison of $16 \mathrm{~S}$ rRNA gene sequences that were at least $90 \%$ complete. Bootstrap values $>50 \%$ (based on 1000 replications) are given at branch points. Bar, 0.01 substitutions per nucleotide position. 
Table 1. Phenotypic characteristics of strain $\mathrm{HY}-50 \mathrm{R}^{\top}$ and representatives of related genera of the family Chitinophagaceae

Taxa: 1, strain HY-50R ${ }^{\mathrm{T}}$; 2, Ferruginibacter; 3, Filimonas; 4, Flavihumibacter; 5, Flavisolibacterium; 6, Hydrotalea; 7, Lacibacter; 8, Niabella; 9, Niastella; 10, Parasegetibacter; 11, Sediminibacterium; 12, Segetibacter; 13, Terrimonas. Data in columns 4 and 6 from Zhang et al. (2010) and Kämpfer et al. (2011), respectively. All other data are from this study except for that of the type strain Terrimonas aquatica RIB1-6 ${ }^{\mathrm{T}}$ (Sheu et al., 2010). +, All species positive; -, all species negative; w, all species weakly positive; v, variable among species; ND, no data available.

\begin{tabular}{|c|c|c|c|c|c|c|c|c|c|c|c|c|c|}
\hline Characteristic & 1 & 2 & 3 & 4 & 5 & 6 & 7 & 8 & 9 & 10 & 11 & 12 & 13 \\
\hline Gliding motility & - & - & + & - & - & - & + & - & $\mathrm{v}$ & + & + & - & - \\
\hline Gram-reaction & - & - & - & + & - & - & - & - & - & - & - & - & - \\
\hline Growth at $37^{\circ} \mathrm{C}$ & + & - & - & + & $\mathrm{v}$ & + & + & - & $\mathrm{v}$ & + & + & - & + \\
\hline \multicolumn{14}{|l|}{ Hydrolysis of: } \\
\hline Chitin & - & - & - & $\mathrm{ND}$ & - & $\mathrm{ND}$ & - & - & $\mathrm{v}$ & - & - & - & - \\
\hline Casein & - & - & - & + & - & $\mathrm{ND}$ & + & $\mathrm{v}$ & + & - & - & - & $\mathrm{v}$ \\
\hline Starch & - & - & - & - & - & ND & + & $\mathrm{v}$ & - & + & - & - & $\mathrm{v}$ \\
\hline Gelatin & - & + & + & + & + & $\mathrm{ND}$ & + & $\mathrm{v}$ & + & + & + & - & $\mathrm{v}$ \\
\hline L-Arginine & - & - & - & + & - & ND & - & - & - & - & - & - & - \\
\hline \multicolumn{14}{|l|}{ Production of: } \\
\hline Flexirubin-type pigments & - & - & - & ND & - & - & - & + & - & - & - & - & $\mathrm{V}$ \\
\hline Indole & - & - & - & ND & - & $\mathrm{ND}$ & - & $\mathrm{v}$ & - & - & - & - & - \\
\hline Oxidase & - & + & + & $\mathrm{w}$ & $\mathrm{v}$ & + & + & $\mathrm{v}$ & $\mathrm{v}$ & + & + & + & + \\
\hline Catalase & + & + & + & + & $\mathrm{v}$ & + & + & + & - & + & + & + & + \\
\hline$\beta$-Galactosidase & + & $\mathrm{v}$ & + & $\mathrm{ND}$ & + & + & + & + & + & + & + & - & + \\
\hline \multicolumn{14}{|l|}{ Assimilation of: } \\
\hline D-Glucose & - & - & + & ND & - & - & - & + & $\mathrm{v}$ & + & - & - & + \\
\hline L-Arabinose & - & - & + & + & $\mathrm{v}$ & - & - & + & $\mathrm{v}$ & $\mathrm{w}$ & - & - & + \\
\hline D-Mannose & - & - & + & + & $\mathrm{v}$ & - & - & + & $\mathrm{v}$ & + & - & - & $\mathrm{v}$ \\
\hline D-Mannitol & - & - & - & - & - & - & - & $\mathrm{v}$ & - & - & - & - & - \\
\hline$N$-Acetylglucosamine & - & - & - & ND & $\mathrm{v}$ & - & + & + & - & + & - & - & $\mathrm{V}$ \\
\hline Maltose & - & - & + & + & $\mathrm{v}$ & - & + & + & $\mathrm{v}$ & + & - & - & + \\
\hline Potassium gluconate & - & - & - & - & $\mathrm{v}$ & - & - & $\mathrm{v}$ & - & - & - & - & - \\
\hline Trisodium citrate & - & - & - & $\mathrm{ND}$ & - & - & - & $\mathrm{v}$ & - & - & - & - & - \\
\hline
\end{tabular}

(Fig. 1) indicated that strain $\mathrm{HY}-50 \mathrm{R}^{\mathrm{T}}$ belonged to the family Chitinophagaceae. The closest relatives of strain HY-50R ${ }^{\mathrm{T}}$ were Niastella populi THYL- $44^{\mathrm{T}} \quad(93.6 \% 16 \mathrm{~S}$ rRNA gene sequence similarity), Flavisolibacter ginsengisoli Gsoil $643^{\mathrm{T}}(93.5 \%)$, Terrimonas ferruginea IAM $15098^{\mathrm{T}}$ $(93.3 \%)$ and Flavisolibacter ginsengiterrae Gsoil $492^{\mathrm{T}}$ $(93.2 \%)$. The phylogenetic tree reconstructed using the maximum-parsimony method (Supplementary Fig. S1, available in IJSEM Online) was similar to that reconstructed using the neighbour-joining method. Analysis of the DNA showed that the $\mathrm{G}+\mathrm{C}$ content of strain $\mathrm{HY}-50 \mathrm{R}^{\mathrm{T}}$ was $46.8 \mathrm{~mol} \%$.

Strain HY-50R ${ }^{\mathrm{T}}$ was oxidase-negative and catalase-positive. Cells of the isolate were Gram-reaction-negative and $0.2-0.4 \times 1.7-2 \mu \mathrm{m}$. Growth of the strain occurred at 15$37{ }^{\circ} \mathrm{C}$, at $\mathrm{pH} 5-8$ and in $0-2 \%(\mathrm{w} / \mathrm{v}) \mathrm{NaCl}$. Yellowcoloured flexirubin-like pigment was not produced. Other phenotypic features of strain $\mathrm{HY}-50 \mathrm{R}^{\mathrm{T}}$ are summarized in the species description. Strain $\mathrm{HY}-50 \mathrm{R}^{\mathrm{T}}$ exhibited some phenotypic differences when compared with other representatives of the family Chitinophagaceae (Table 1).
Furthermore, several characteristics, such as the inability to degrade gelatin and the presence of $\alpha$-mannosidase activity, distinguished strain $\mathrm{HY}-50 \mathrm{R}^{\mathrm{T}}$ from its closest phylogenetic neighbours. Differential characteristics of strain $\mathrm{HY}-50 \mathrm{R}^{\mathrm{T}}$ and closely related type strains are shown in Table 2.

Menaquinone 7 (MK-7) was detected as the major respiratory quinone of strain $\mathrm{HY}-50 \mathrm{R}^{\mathrm{T}}$, which is characteristic of species of the family Chitinophagaceae. The polyamine pattern showed a predominance of homospermidine. Strain $\mathrm{HY}-50 \mathrm{R}^{\mathrm{T}}$ possessed iso- $\mathrm{C}_{15: 1} \mathrm{G}(11.7 \%)$, iso- $\mathrm{C}_{15: 0}(19.6 \%)$ and iso- $\mathrm{C}_{17: 0} 3-\mathrm{OH}(19.3 \%)$ as the major fatty acids. In addition, iso- $\mathrm{C}_{13: 0}(6.1 \%)$, summed feature $1\left(\mathrm{C}_{13: 0} 3-\mathrm{OH}\right.$ and/or iso- $\left.\mathrm{C}_{15: 1} \mathrm{H}, 5.6 \%\right)$ and summed feature 3 (iso- $\mathrm{C}_{15: 0} 2-\mathrm{OH}$ and/or $\mathrm{C}_{16: 1} \omega 7 c$, $6.9 \%)$ were produced in moderate amounts. Fatty acid profiles of strain $\mathrm{HY}-50 \mathrm{R}^{\mathrm{T}}$ and the type strains of related species are shown in Table 3 . The presence of a relatively high proportion of iso- $\mathrm{C}_{13: 0}(6.1 \%)$ distinguished strain $\mathrm{HY}-50 \mathrm{R}^{\mathrm{T}}$ from recognized species of phylogenetically related genera. Strain $\mathrm{HY}-50 \mathrm{R}^{\mathrm{T}}$ could also be differentiated 
Table 2. Phenotypic characteristics of strain $H Y-50 R^{\top}$ and closely related type strains of members of the family Chitinophagaceae

Taxa: 1, strain $\mathrm{HY}^{-50 \mathrm{R}^{\mathrm{T}}}$; 2, Flavisolibacterium ginsengisoli Gsoil $643^{\mathrm{T}} ; 3$, Flavisolibacterium ginsengiterrae Gsoil $492^{\mathrm{T}}$; 4, Niastella populi THYL-44 ${ }^{\mathrm{T}}$; 5, Terrimonas ferruginea DSM $30193^{\mathrm{T}}$. All data from this study. + , Positive; -, negative; w, weakly positive.

\begin{tabular}{|lccccc|}
\hline Characteristic & $\mathbf{1}$ & $\mathbf{2}$ & $\mathbf{3}$ & $\mathbf{4}$ & $\mathbf{5}$ \\
\hline Growth on TSA & + & - & - & + & + \\
Tolerance of NaCl (\%, w/v) & 2.0 & 1.0 & 3.0 & 1.0 & 1.0 \\
Enzyme activities: & & & & & \\
$\quad$ Esterase (C4), esterase lipase (C8) & + & + & + & - & + \\
$\alpha$-Chymotrypsin & - & + & + & - & - \\
$\alpha$-Galactosidase & + & + & + & - & - \\
$\alpha$-Glucosidase & + & + & + & - & w \\
$\beta$-Glucosidase & + & w & + & - & - \\
$\alpha$-Mannosidase & + & - & - & - & - \\
Assimilation of D-Glucose, & - & - & - & & + \\
L-arabinose, D-mannose, maltose & & & & & \\
\end{tabular}

from the type species of its closest relatives by the absence of iso- $\mathrm{C}_{15: 0} \quad 3-\mathrm{OH}$ and $\mathrm{C}_{18: 0}$ and by some other quantitative differences between fatty acid compositions.

Based on the phenotypic, chemotaxonomic and genetic data and the relatively low levels of 16S rRNA gene sequence similarity between strain $\mathrm{HY}-50 \mathrm{R}^{\mathrm{T}}$ and members of related genera of the family Chitinophagaceae, strain $\mathrm{HY}-50 \mathrm{R}^{\mathrm{T}}$ represents a novel species of a novel genus in the family Chitinophagaceae, for which the name Flavitalea populi gen. nov., sp. nov. is proposed.

\section{Description of Flavitalea gen. nov.}

Flavitalea (Fla.vi.ta'le.a. L. adj. flavus yellow; L. fem. n. talea a slender staff, a rod; N.L. fem. n. Flavitalea a yellowcoloured rod).

Cells are Gram-reaction-negative, strictly aerobic, rodshaped and non-motile. Oxidase-negative and catalasepositive. MK-7 is the major respiratory quinone. Flexirubinlike pigments are not detected. Homospermidine is the major polyamine component. Phylogenetically positioned within the family Chitinophagaceae, phylum Bacteroidetes. The type species is Flavitalea populi.

\section{Description of Flavitalea populi sp. nov.}

Flavitalea populi (po'pu.li. L. gen. n. populi of a poplar, pertaining to Populus, the genus name of the poplar trees that grow in the forest the strain was isolated from).

Cells are $0.2-0.4 \times 1.7-2 \mu \mathrm{m}$ in size. Colonies are circular, convex, smooth, opaque and yellow on $0.1 \times$ TSA. Grows on NA, R2A, TSA and $0.1 \times$ TSA but not on MacConkey's agar at $30{ }^{\circ} \mathrm{C}$. Grows at $15-37{ }^{\circ} \mathrm{C}$ (optimum $30{ }^{\circ} \mathrm{C}$ ) and pH 5-8 (optimum pH 7). Tolerates up to $2 \%(w / v) ~ N a C l$. Hydrolyses aesculin but not gelatin, starch, casein, chitin or cellulose. Nitrate is not reduced to nitrite and hydrogen sulfide is not produced. Positive for $\mathrm{N}$-acetyl-D-glucosamine, sucrose, maltose, D-glucose, salicin and melibiose assimilation. Negative for citrate utilization, indole production, glucose acidification, arginine dihydrolase and urease activities and assimilation of L-arabinose, Dmannose, D-mannitol, potassium gluconate, capric acid, adipic acid, malic acid, trisodium citrate, phenylacetic acid, L-rhamnose, D-ribose, myo-inositol, itaconic acid, suberic acid, sodium malonate, sodium acetate, lactic acid, Lalanine, potassium 5-ketogluconate, glycogen, 3-hydroxybenzoic acid, L-serine, D-mannitol, L-fucose, D-sorbitol, propionic acid, valeric acid, L-histidine, 3-hydroxybutyric acid, 4-hydroxybenzoic acid, potassium 2-ketogluconate and L-proline. In the API ZYM system, positive for alkaline phosphatase, esterase (C4), esterase lipase (C8), leucine arylamidase, valine arylamidase, cystine arylamidase, acid phosphatase, naphthol-AS-B1-phosphohydrolase, $\alpha$ - and $\beta$-galactosidase, $\alpha$ - and $\beta$-glucosidase, $N$-acetyl- $\beta$-glucosaminidase and $\alpha$-mannosidase activities but negative for lipase (C14), trypsin, $\alpha$-chymotrypsin, $\beta$-glucuronidase and $\alpha$-fucosidase activities. The major fatty acids are iso- $C_{15: 1}$ $\mathrm{G}$, iso- $\mathrm{C}_{15: 0}$ and iso- $\mathrm{C}_{17: 0} 3-\mathrm{OH}$.

The type strain, HY-50R ${ }^{\mathrm{T}}$ (=CCTCC AB $208255^{\mathrm{T}}=\mathrm{NRRL}$ B-59222 ${ }^{\mathrm{T}}$ ), was isolated from a soil sample from a Euphrates poplar forest in Xinjiang, China. The DNA $\mathrm{G}+\mathrm{C}$ content of type strain is $46.8 \mathrm{~mol} \%$.

\section{Acknowledgements}

We would like to thank J. P. Euzéby for checking the species epithet for correctness. This work was supported by the R \& D Infrastructure and Facility Development Program from the Ministry of Science and Technology of the People's Republic of China (grant no. 2005DKA21208). 
Table 3. Cellular fatty acid compositions (\%) of strain $\mathrm{HY}-50 \mathrm{R}^{\top}$ and closely related type strains of members of the family Chitinophagaceae

Taxa: 1, strain $\mathrm{HY}^{-50 \mathrm{R}^{\mathrm{T}}} ; 2$, Ferruginibacter alkalilentus $\mathrm{HU} 1-\mathrm{GD} 23^{\mathrm{T}} ; 3$, Ferruginibacter lapsinanis $\mathrm{HU} 1-\mathrm{HG}_{2} 2^{\mathrm{T}} ; 4$, Filimonas lacunae $\mathrm{YT}^{\mathrm{T}} 1^{\mathrm{T}} ; 5$, Flavihumibacter petaseus $\mathrm{T}^{\mathrm{T}} 1^{\mathrm{T}} ; 6$, Flavisolibacterium ginsengisoli Gsoil $643^{\mathrm{T}} ;$; , Flavisolibacterium ginsengiterrae Gsoil $492^{\mathrm{T}} ; 8$, Hydrotalea flava CCUG $51397^{\mathrm{T}} ; 9$, Lacibacter cauensis $\mathrm{NJ}-8^{\mathrm{T}} ; 10$, Niabella aurantiaca R2A15-11 ${ }^{\mathrm{T}} ; 11$, Niabella soli JS13-8 ${ }^{\mathrm{T}}$;

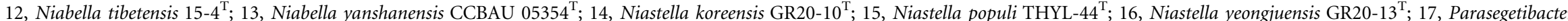
luojiensis RHYL-37 $7^{\mathrm{T}} ; 18$, Sediminibacterium salmoneum $\mathrm{NJ}-44^{\mathrm{T}} ; 19$, Segetibacter koreensis Gsoil $664^{\mathrm{T}} ; 20$, Terrimonas ferruginea DSM $30193^{\mathrm{T}} ; 21$, Terrimonas lutea $\mathrm{DY}^{\mathrm{T}}$. Data in columns 5 and 8 were taken from Zhang et al. (2010) and Kämpfer et al. (2011), respectively. Values are percentages of total fatty acids; fatty acids amounting to $<1 \%$ of total fatty acids in all strains studied are not listed; tr, $\operatorname{trace}(<1 \%)$.

\begin{tabular}{|c|c|c|c|c|c|c|c|c|c|c|c|c|c|c|c|c|c|c|c|c|c|}
\hline Fatty acid & 1 & 2 & 3 & 4 & 5 & 6 & 7 & 8 & 9 & 10 & 11 & 12 & 13 & 14 & 15 & 16 & 17 & 18 & 19 & 20 & 21 \\
\hline iso- $\mathrm{C}_{13: 0}$ & 6.1 & $\operatorname{tr}$ & 2.5 & $\operatorname{tr}$ & & $\operatorname{tr}$ & $\operatorname{tr}$ & 4.2 & $\operatorname{tr}$ & $\operatorname{tr}$ & & $\operatorname{tr}$ & & $\operatorname{tr}$ & $\operatorname{tr}$ & $\operatorname{tr}$ & $\operatorname{tr}$ & $\operatorname{tr}$ & $\operatorname{tr}$ & $\operatorname{tr}$ & $\operatorname{tr}$ \\
\hline iso- $\mathrm{C}_{14: 0}$ & $\operatorname{tr}$ & $\operatorname{tr}$ & & $\operatorname{tr}$ & & $\operatorname{tr}$ & 2.5 & & $\operatorname{tr}$ & & & $\operatorname{tr}$ & $\operatorname{tr}$ & $\operatorname{tr}$ & $\operatorname{tr}$ & $\operatorname{tr}$ & $\operatorname{tr}$ & 1.3 & 6.3 & $\operatorname{tr}$ & $\operatorname{tr}$ \\
\hline $\mathrm{C}_{14: 0}$ & & 1.9 & 1.2 & 2.4 & & & & & 1.1 & 1.1 & 2.7 & 1.3 & 1.4 & & & $\operatorname{tr}$ & 2.1 & 1.9 & 1.3 & 1.6 & 1.6 \\
\hline iso- $\mathrm{C}_{13: 0} 3-\mathrm{OH}$ & 4.8 & $\operatorname{tr}$ & $\operatorname{tr}$ & $\operatorname{tr}$ & & 1.9 & 1.3 & & $\operatorname{tr}$ & & 1.5 & $\operatorname{tr}$ & $\operatorname{tr}$ & $\operatorname{tr}$ & $\operatorname{tr}$ & $\operatorname{tr}$ & 5.2 & 1.6 & $\operatorname{tr}$ & $\operatorname{tr}$ & $\operatorname{tr}$ \\
\hline iso- $\mathrm{C}_{15: 1} \mathrm{G}$ & 11.7 & 15.2 & 16.0 & 18.7 & 13.3 & 5.4 & 7.0 & 8.2 & 10.2 & 21.8 & 18.5 & 13.7 & 26.4 & 15.8 & 17.4 & 14.8 & 14.7 & 17.7 & & 23.7 & 23.4 \\
\hline iso- $\mathrm{C}_{15: 1} \mathrm{~F}$ & & & & & & & & & & & & & & & & & & & $\operatorname{tr}$ & & \\
\hline anteiso- $\mathrm{C}_{15: 1} \mathrm{~A}$ & 2.9 & $\operatorname{tr}$ & $\operatorname{tr}$ & $\operatorname{tr}$ & & 3.5 & 5.4 & & $\operatorname{tr}$ & $\operatorname{tr}$ & $\operatorname{tr}$ & $\operatorname{tr}$ & $\operatorname{tr}$ & 1.0 & 1.3 & $\operatorname{tr}$ & 3.7 & 4.4 & & $\operatorname{tr}$ & $\operatorname{tr}$ \\
\hline iso- $\mathrm{C}_{15: 0}$ & 19.6 & 18.4 & 32.5 & 23.7 & 33.8 & 24.3 & 25.7 & 34.8 & 33.6 & 30.6 & 28.6 & 42.9 & 29.0 & 23.3 & 28.6 & 29.1 & 19.5 & 17.6 & 15.3 & 27.6 & 20.8 \\
\hline anteiso- $\mathrm{C}_{15: 0}$ & 4.6 & 2.1 & 1.3 & $\operatorname{tr}$ & & 9.2 & 9.7 & 1.7 & 1.1 & 1.8 & $\operatorname{tr}$ & 1.3 & $\operatorname{tr}$ & 4.5 & 4.9 & 5.5 & 3.3 & 10.6 & 21.1 & 2.4 & 1.4 \\
\hline $\mathrm{C}_{15: 0}$ & 1. 2 & 4.2 & $\operatorname{tr}$ & $\operatorname{tr}$ & 8.0 & 1.0 & 1.6 & $\operatorname{tr}$ & 4.3 & 8.5 & 1.6 & 3.2 & 1.6 & 2.7 & $\operatorname{tr}$ & 1.9 & 2.7 & $\operatorname{tr}$ & 2.9 & 6.6 & 5.7 \\
\hline iso- $\mathrm{C}_{16: 1} \mathrm{H}$ & & & & & & 1.1 & 2.3 & & & & & & & & & & & & 3.0 & & \\
\hline iso- $\mathrm{C}_{16: 0}$ & $\operatorname{tr}$ & 1.2 & $\operatorname{tr}$ & 1.7 & & 1.8 & 4.2 & $\operatorname{tr}$ & 2.1 & $\operatorname{tr}$ & & $\operatorname{tr}$ & 2.3 & $\operatorname{tr}$ & $\operatorname{tr}$ & $\operatorname{tr}$ & 3.0 & 1.7 & 13.3 & 1.0 & $\operatorname{tr}$ \\
\hline $\mathrm{C}_{16: 1} \omega 5 c$ & 2.5 & & & 1.8 & 5.8 & $\operatorname{tr}$ & $\operatorname{tr}$ & & 2.2 & & & & 2.4 & & & 1.1 & & $\operatorname{tr}$ & 3.1 & & \\
\hline$C_{16: 0}$ & 2.0 & 7.7 & 6.9 & 4.9 & 2.2 & 8.2 & 4.2 & $\operatorname{tr}$ & 4.4 & 5.7 & 9.8 & 3.9 & 3.1 & 4.8 & 3.9 & 2.9 & 10.1 & 5.0 & 1.5 & 5.2 & 3.5 \\
\hline iso- $\mathrm{C}_{15: 0} 3-\mathrm{OH}$ & & 11.0 & 3.3 & 4.0 & 3.9 & $\operatorname{tr}$ & 1.1 & 4.0 & 3.2 & 2.5 & 2.3 & 2.8 & 2.8 & 1.3 & 1.6 & 2.1 & & 4.2 & 1.2 & 1.8 & 1.6 \\
\hline $\mathrm{C}_{15: 0} 2-\mathrm{OH}$ & $\operatorname{tr}$ & 1.4 & $\operatorname{tr}$ & & & $\operatorname{tr}$ & $\operatorname{tr}$ & $\operatorname{tr}$ & $\operatorname{tr}$ & 1.3 & & $\operatorname{tr}$ & & 1.0 & $\operatorname{tr}$ & $\operatorname{tr}$ & $\operatorname{tr}$ & 1.2 & 1.0 & $\operatorname{tr}$ & 2.7 \\
\hline $\mathrm{C}_{15: 0} 3-\mathrm{OH}$ & & 1.2 & & & & & & & $\operatorname{tr}$ & & & $\operatorname{tr}$ & & $\operatorname{tr}$ & & $\operatorname{tr}$ & & & $\operatorname{tr}$ & $\operatorname{tr}$ & 1.1 \\
\hline iso- $\mathrm{C}_{17: 0}$ & 4.1 & & $\operatorname{tr}$ & $\operatorname{tr}$ & & 2.6 & $\operatorname{tr}$ & & 1.3 & & & $\operatorname{tr}$ & $\operatorname{tr}$ & $\operatorname{tr}$ & $\operatorname{tr}$ & 1.1 & 1.1 & & $\operatorname{tr}$ & $\operatorname{tr}$ & \\
\hline anteiso- $\mathrm{C}_{17: 0}$ & $\operatorname{tr}$ & $\operatorname{tr}$ & $\operatorname{tr}$ & & & $\operatorname{tr}$ & $\operatorname{tr}$ & & $\operatorname{tr}$ & $\operatorname{tr}$ & & & $\operatorname{tr}$ & $\operatorname{tr}$ & $\operatorname{tr}$ & 2.3 & $\operatorname{tr}$ & & $\operatorname{tr}$ & $\operatorname{tr}$ & \\
\hline$C_{17: 1} \omega 6 c$ & 1.2 & $\operatorname{tr}$ & $\operatorname{tr}$ & & & 1.9 & 6.3 & & $\operatorname{tr}$ & & & & 1.0 & $\operatorname{tr}$ & & $\operatorname{tr}$ & $\operatorname{tr}$ & & 1.1 & & $\operatorname{tr}$ \\
\hline $\mathrm{C}_{17: 0}$ & & $\operatorname{tr}$ & $\operatorname{tr}$ & & & $\operatorname{tr}$ & & & & & $\operatorname{tr}$ & & & $\operatorname{tr}$ & $\operatorname{tr}$ & $\operatorname{tr}$ & $\operatorname{tr}$ & & & & \\
\hline iso- $\mathrm{C}_{16: 0} 3-\mathrm{OH}$ & $\operatorname{tr}$ & 6.1 & $\operatorname{tr}$ & 1.1 & & $\operatorname{tr}$ & $\operatorname{tr}$ & 1.2 & $\operatorname{tr}$ & $\operatorname{tr}$ & & $\operatorname{tr}$ & $\operatorname{tr}$ & 1.1 & $\operatorname{tr}$ & $\operatorname{tr}$ & $\operatorname{tr}$ & 6.3 & 11.5 & $\operatorname{tr}$ & $\operatorname{tr}$ \\
\hline $\mathrm{C}_{16: 0} 3-\mathrm{OH}$ & $\operatorname{tr}$ & 3.5 & 2.5 & 5.1 & 2.7 & $\operatorname{tr}$ & $\operatorname{tr}$ & $\operatorname{tr}$ & 1.3 & 2.4 & 1.8 & 3.4 & 1.7 & 1.4 & 1.6 & 1.7 & 1.8 & 7.1 & 1.3 & 2.9 & 5.1 \\
\hline $\mathrm{C}_{18: 1} \omega 9 c$ & & $\operatorname{tr}$ & $\operatorname{tr}$ & & & 1.5 & $\operatorname{tr}$ & & & & 3.9 & & & $\operatorname{tr}$ & $\operatorname{tr}$ & & 1.9 & & $\operatorname{tr}$ & $\operatorname{tr}$ & $\operatorname{tr}$ \\
\hline $\mathrm{C}_{18: 0}$ & & 3.7 & 1.3 & & & 3.7 & $\operatorname{tr}$ & & $\operatorname{tr}$ & 1.7 & 6.8 & $\operatorname{tr}$ & $\operatorname{tr}$ & 2.4 & 1.9 & $\operatorname{tr}$ & 3.9 & $\operatorname{tr}$ & 1.1 & 1.8 & $\operatorname{tr}$ \\
\hline iso- $\mathrm{C}_{17: 0} 3-\mathrm{OH}$ & 19.3 & 12.3 & 15.4 & 16.4 & 12.9 & 10.6 & 8.1 & 16.9 & 21.8 & 14.8 & 8.6 & 12.0 & 14.4 & 25.9 & 23.9 & 23.4 & 16.3 & 11.4 & 3.6 & 16.8 & 12.7 \\
\hline $\mathrm{C}_{17: 0} 2-\mathrm{OH}$ & $\operatorname{tr}$ & 1.2 & $\operatorname{tr}$ & $\operatorname{tr}$ & & $\operatorname{tr}$ & $\operatorname{tr}$ & $\operatorname{tr}$ & $\operatorname{tr}$ & $\operatorname{tr}$ & & $\operatorname{tr}$ & $\operatorname{tr}$ & 2.9 & 1.8 & 2.3 & 1.3 & 3.6 & 2.1 & $\operatorname{tr}$ & $\operatorname{tr}$ \\
\hline $\mathrm{C}_{17: 0} 3-\mathrm{OH}$ & $\operatorname{tr}$ & $\operatorname{tr}$ & & & 1.1 & & $\operatorname{tr}$ & & $\operatorname{tr}$ & $\operatorname{tr}$ & & $\operatorname{tr}$ & & 1.5 & $\operatorname{tr}$ & $\operatorname{tr}$ & $\operatorname{tr}$ & & $\operatorname{tr}$ & 1.0 & 1.5 \\
\hline Unknown 13.565 & 1.9 & $\operatorname{tr}$ & $\operatorname{tr}$ & 2.8 & 1.5 & $\operatorname{tr}$ & $\operatorname{tr}$ & 8.4 & $\operatorname{tr}$ & & $\operatorname{tr}$ & $\operatorname{tr}$ & $\operatorname{tr}$ & $\operatorname{tr}$ & $\operatorname{tr}$ & $\operatorname{tr}$ & & $\operatorname{tr}$ & $\operatorname{tr}$ & & $\operatorname{tr}$ \\
\hline Unknown 14.959 & & & & & & & & & & & & & & & & & & & 1.6 & & \\
\hline Unknown 16.582 & 1.9 & $\operatorname{tr}$ & 1.1 & 1.0 & & 1.0 & $\operatorname{tr}$ & 1.7 & 1.7 & 1.9 & 1.0 & 1.0 & 1.1 & 1.9 & 2.9 & 1.9 & 1.2 & $\operatorname{tr}$ & $\operatorname{tr}$ & 1.3 & 1.0 \\
\hline
\end{tabular}




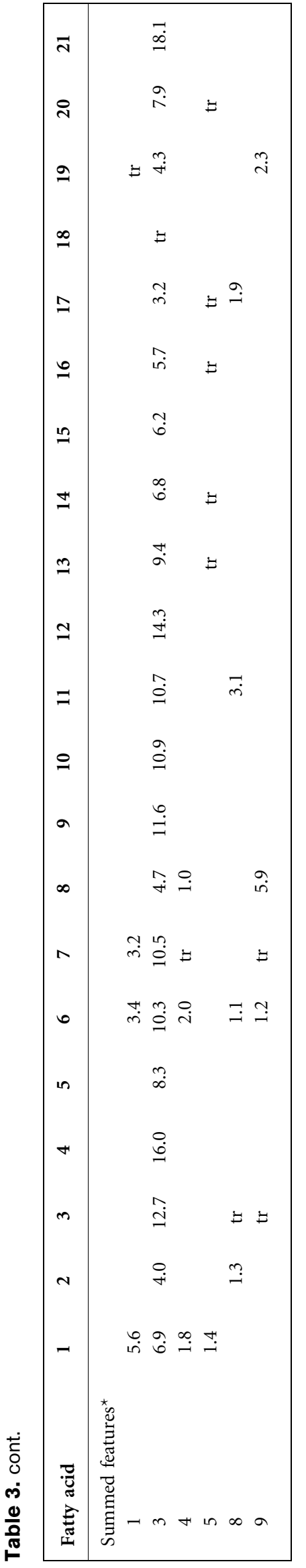

\section{References}

An, D. S., Lee, H. G., Im, W. T., Liu, Q. M. \& Lee, S. T. (2007). Segetibacter koreensis gen. nov., sp. nov., a novel member of the phylum Bacteroidetes, isolated from the soil of a ginseng field in South Korea. Int J Syst Evol Microbiol 57, 1828-1833.

Choi, D. H., Zhang, G. I., Noh, J. H., Kim, W. S. \& Cho, B. C. (2009). Gracilimonas tropica gen. nov., sp. nov., isolated from a Synechococcus culture. Int J Syst Evol Microbiol 59, 1167-1172.

Chun, J., Lee, J.-H., Jung, Y., Kim, M., Kim, S., Kim, B. K. \& Lim, Y. W. (2007). EzTaxon: a web-based tool for the identification of prokaryotes based on 16S ribosomal RNA gene sequences. Int J Syst Evol Microbiol 57, 2259-2261.

Felsenstein, J. (1985). Confidence limits on phylogenies: an approach using the bootstrap. Evolution 39, 783-791.

Fitch, W. M. (1971). Towards defining the course of evolution: minimum change for a specific tree topology. Syst Zool 20, 406-416.

Garrity, G. M. \& Holt, J. G. (2001). The road map to the Manual. In Bergey's Manual of Systematic Bacteriology, 2nd edn, vol. 1, pp. 119166. Edited by D. R. Boone, R. W. Castenholz \& G. M. Garrity. New York: Springer.

Gerhardt, P., Murray, R. G. E., Wood, W. A. \& Krieg, N. R. (editors) (1994). Methods for General and Molecular Bacteriology. Washington, DC: American Society for Microbiology.

Güde, H. (1980). Occurrence of cytophagas in sewage plants. Appl Environ Microbiol 39, 756-763.

Kämpfer, P., Young, C. C., Sridhar, K. R., Arun, A. B., Lai, W. A., Shen, F. T. \& Rekha, P. D. (2006). Transfer of [Flexibacter] sancti, [Flexibacter] filiformis, [Flexibacter] japonensis and [Cytophaga] arvensicola to the genus Chitinophaga and description of Chitinophaga skermanii sp. nov. Int J Syst Evol Microbiol 56, 2223-2228.

Kämpfer, P., Lodders, N. \& Falsen, E. (2011). Hydrotalea flava gen. nov. sp. nov., a new species of the phylum Bacteriodetes and allocation of the genera Chitinophaga, Sediminibacterium, Lacibacter, Flavihumibacter, Flavisolibacter, Niabella, Niastella, Segetibacter, Parasegetibacter, Terrimonas, Ferruginibacter, Filimonas and Hydrotalea to the family Chitinophagaceae fam. nov. Int J Syst Evol Microbiol 61, 518-523.

Kim, B. Y., Weon, H. Y., Yoo, S. H., Hong, S. B., Kwon, S. W., Stackebrandt, E. \& Go, S. J. (2007). Niabella aurantiaca gen. nov., sp. nov., isolated from a greenhouse soil in Korea. Int J Syst Evol Microbiol 57, 538-541.

Kimura, M. (1980). A simple method for estimating evolutionary rates of base substitutions through comparative studies of nucleotide sequences. J Mol Evol 16, 111-120.

Lane, D. J. (1991). 16S/23S rRNA sequencing. In Nucleic Acid Techniques in Bacterial Systematics, pp. 115-147. Edited by E. Stackebrandt \& M. Goodfellow. Chichester: Wiley.

Lim, J. H., Baek, S.-H. \& Lee, S.-T. (2009). Ferruginibacter alkalilentus gen. nov., sp. nov. and Ferruginibacter lapsinanis sp. nov., novel members of the family 'Chitinophagaceae' in the phylum Bacteroidetes, isolated from freshwater sediment. Int J Syst Bacteriol 59, 2394-2399.

Mesbah, M., Premachandran, U. \& Whitman, W. B. (1989). Precise measurement of the $\mathrm{G}+\mathrm{C}$ content of deoxyribonucleic acid by highperformance liquid chromatography. Int J Syst Bacteriol 39, 159-167.

MIDI (1999). Sherlock microbial identification system, operating manual, version 3.0. Newark, DE: MIDI, Inc.

Qu, J. H. \& Yuan, H. L. (2008). Sediminibacterium salmoneum gen. nov., sp. nov., a member of the phylum Bacteroidetes isolated from sediment of a eutrophic reservoir. Int J Syst Evol Microbiol 58, 2191-2194.

Qu, J. H., Yuan, H. L., Yang, J. S., Li, H. F. \& Chen, N. (2009). Lacibacter cauensis gen. nov., sp. nov., a novel member of the phylum 
Bacteroidetes isolated from sediment of a eutrophic lake. Int J Syst Evol Microbiol 59, 1153-1157.

Saitou, N. \& Nei, M. (1987). The neighbor-joining method: a new method for reconstructing phylogenetic trees. Mol Biol Evol 4, 406-425.

Schenkel, E., Berlaimont, V., Dubois, J., Helson-Cambier, M. \& Hanocq, M. (1995). Improved high-performance liquid chromatographic method for the determination of polyamines as their benzoylated derivatives: application to P388 cancer cells. J Chromatogr B Biomed Appl 668, 189-197.

Sheu, S.-Y., Cho, N.-T., Arun, A. B. \& Chen, W.-M. (2010). Terrimonas aquatica sp. nov., isolated from a freshwater spring. Int J Syst Evol Microbiol 60, 2705-2709.

Shiratori, H., Tagami, Y., Morishita, T., Kamihara, Y., Beppu, T. \& Ueda, K. (2009). Filimonas lacunae gen. nov., sp. nov., a member of the phylum Bacteroidetes isolated from fresh water. Int J Syst Evol Microbiol 59, 1137-1142.

Smibert, R. M. \& Krieg, N. R. (1994). Phenotypic characterization. In Methods for General and Molecular Bacteriology, pp. 607-654. Edited by P. Gerhardt, R. G. E. Murray, W. A. Woods \& N. R. Krieg. Washington, DC: American Society for Microbiology.

Tamura, K., Dudley, J., Nei, M. \& Kumar, S. (2007). MEGA4: molecular evolutionary genetics analysis (MEGA) software version 4.0. Mol Biol Evol 24, 1596-1599.

Thompson, J. D., Gibson, T. J., Plewniak, F., Jeanmougin, F. \& Higgins, D. G. (1997). The CLUSTAL_X windows interface: flexible strategies for multiple sequence alignment aided by quality analysis tools. Nucleic Acids Res 25, 4876-4882.

Urios, L., Agogué, H., Lesongeur, F., Stackebrandt, E. \& Lebaron, P. (2006). Balneola vulgaris gen. nov., sp. nov., a member of the phylum Bacteroidetes from the north-western Mediterranean Sea. Int J Syst Evol Microbiol 56, 1883-1887.
Weon, H. Y., Kim, B. Y., Yoo, S. H., Lee, S. Y., Kwon, S. W., Go, S. J. \& Stackebrandt, E. (2006). Niastella koreensis gen. nov., sp. nov. and Niastella yeongjuensis sp. nov., novel members of the phylum Bacteroidetes, isolated from soil cultivated with Korean ginseng. Int J Syst Evol Microbiol 56, 1777-1782.

Wilson, K. (1987). Preparation of genomic DNA from bacteria. In Current Protocols in Molecular Biology, pp. 241-245. Edited by F. M. Ausubel, R. Brent, R. E. Kingston, D. D. Moore, J. G. Seidman, J. A. Smith \& K. Struhl. New York: Greene Publishing and WileyInterscience.

Xie, C. H. \& Yokota, A. (2003). Phylogenetic analyses of Lampropedia hyalina based on the 16S rRNA gene sequence. J Gen Appl Microbiol 49, 345-349.

Xie, C. H. \& Yokota, A. (2006). Reclassification of [Flavobacterium] ferrugineum as Terrimonas ferruginea gen. nov., comb. nov., and description of Terrimonas lutea sp. nov., isolated from soil. Int J Syst Evol Microbiol 56, 1117-1121.

Xu, P., Li, W.-J., Tang, S.-K., Zhang, Y.-Q., Chen, G.-Z., Chen, H.-H., Xu, L.-H. \& Jiang, C.-L. (2005). Naxibacter alkalitolerans gen. nov., sp. nov., a novel member of the family 'Oxalobacteraceae' isolated from China. Int J Syst Evol Microbiol 55, 1149-1153.

Yoon, M. H. \& Im, W. T. (2007). Flavisolibacter ginsengiterrae gen. nov., sp. nov. and Flavisolibacter ginsengisoli sp. nov., isolated from ginseng cultivating soil. Int J Syst Evol Microbiol 57, 1834-1839.

Zhang, K. D., Tang, Y. L., Zhang, L., Dai, J., Wang, Y., Luo, X. S., Liu, M., Luo, G. C. \& Fang, C. X. (2009). Parasegetibacter luojiensis gen. nov., sp. nov., a member of the phylum Bacteroidetes isolated from a forest soil. Int J Syst Evol Microbiol 59, 3058-3062.

Zhang, N. N., Qu, J.-H., Yuan, H.-L., Sun, Y. M. \& Yang, J.-S. (2010). Flavihumibacter petaseus gen. nov., sp. nov., isolated from soil of a subtropical rainforest. Int J Syst Evol Microbiol 60, 16091612. 